\title{
Shallow-underground accelerator sites for nuclear astrophysics: Is the background low enough?
}

\author{
Tamás Szücs ${ }^{1,2}$, Daniel Bemmerer ${ }^{1, a}$, Thomas Cowan ${ }^{1,3}$, Detlev Degering ${ }^{4}$, Zoltán Elekes ${ }^{2,1}$, Zsolt Fülöp ${ }^{2}$, \\ György Gyürky ${ }^{2}$, Arnd Junghans ${ }^{1}$, Matthias Köhler ${ }^{4}$, Michele Marta ${ }^{1, b}$, Ronald Schwengner ${ }^{1}$, Andreas Wagner ${ }^{1}$, \\ and Kai Zuber ${ }^{3}$ \\ 1 Helmholtz-Zentrum Dresden-Rossendorf (HZDR), Dresden, Germany \\ 2 Institute of Nuclear Research of the Hungarian Academy of Sciences (ATOMKI), Debrecen, Hungary \\ 3 Technische Universität Dresden, Dresden, Germany \\ 4 Verein für Kernverfahrenstechnik und Analytik Rossendorf (VKTA), Dresden, Germany
}

Received: 20 July 2011 / Revised: 23 December 2011

Published online: 30 January 2012

(c) The Author(s) 2012. This article is published with open access at Springerlink.com

Communicated by E. Bellotti

\begin{abstract}
In order to reliably estimate the rate of a charged particle induced nuclear reaction in a nonexplosive astrophysical scenario, its cross-section must be measured far below the Coulomb barrier. However, at the corresponding energies the cross-section values are very low, so that the experimental counting rate is dominated by cosmic-ray induced background, even if a suitable anticoincidence shield is applied. This problem can be overcome by performing an accelerator-based experiment in a deep underground site, as has been done with great success at the LUNA 0.4 MV accelerator in Gran Sasso, Italy. Several underground accelerators with higher beam energy are in the planning phase worldwide. All of them are shielded by over $1000 \mathrm{~m}$ of rock, a depth at which cosmic-ray effects are negligible for the purposes of nuclear astrophysics experiments. It is shown here that a combined approach, using a shallow-underground laboratory below $47 \mathrm{~m}$ of rock and an active shield to veto surviving muons in simple detectors, results in a background level that is not far from that of deep underground sites. Data have been obtained using two "traveling" $\gamma-$ detectors. They have been transported both shallow underground, to the Dresden Felsenkeller in Germany, and deep underground, to the Gran Sasso laboratory in Italy. As shallow-underground facilities are more easily accessible than deep-underground ones, the present finding holds the promise of greatly accelerated progress in the field of cross-section measurements for nuclear astrophysics.
\end{abstract}

\section{Introduction}

Many nuclear physics inputs are needed for the modeling of astrophysical scenarios [1-3], and progress must be made on two frontiers: Nuclei far from the valley of $\beta$ stability will become accessible at next generation radioactive ion beam facilities. For reactions of stable nuclei, the frontier is given by the lack of precise cross-section data at low energy.

These reactions are generally well studied at high energies, above the Coulomb barrier. However, the astrophysically relevant energy range lies far lower. Since extrapolations are fraught with uncertainty, it is desirable to measure the cross-section directly at the relevant energy, or at least close to it. There, the cross-section is very low,

\footnotetext{
a e-mail: d.bemmerer@hzdr.de

b Present address: GSI Helmholtzzentrum für Schwerionenforschung, Darmstadt, Germany
}

so low that the laboratory background counting rate in a detector becomes a limiting factor.

This limitation can be overcome by placing the entire laboratory in a low-background setting underground. Rock overburdens of a few meters thickness suppress the nucleonic component of the cosmic-ray induced background to negligible levels [4]. However, the muonic component is only slowly attenuated when proceeding to greater depth [5]. Muons produce neutrons inside the shielding or the detector itself [6], giving rise to a background that is difficult to suppress. In order for the muon flux to become negligible, depths of $1000 \mathrm{~m}$ of rock or more are required [7]. This depth has been selected with great success by the Laboratory for Underground $\mathrm{Nu}$ clear Astrophysics (LUNA). LUNA is placed in the Gran Sasso laboratory in Italy, below $1400 \mathrm{~m}$ of rock, and with uniquely low background [8-10]. It hosted first a $50 \mathrm{kV}$ accelerator and now a $0.4 \mathrm{MV}$ single-ended accelerator for ${ }^{1} \mathrm{H}^{+}$and ${ }^{4} \mathrm{He}^{+}$ions [11]. 
In recent years, underground nuclear astrophysics experiments have helped put the understanding of nuclear fusion in our Sun on firm experimental ground [12-17]. For further progress, new data at higher energies are needed. A recent expert forum on solar fusion cross-sections called for new data, e.g., on the ${ }^{3} \mathrm{He}(\alpha, \gamma){ }^{7} \mathrm{Be}$ reaction. The same forum also highlighted the need for a higher-energy underground accelerator [18].

Several astrophysical scenarios other than the Sun require new and precise cross-section data for their modeling, as well. This is the case for the reactions of Big Bang nucleosynthesis [19]. In stars heavier than the Sun, hydrogen burning proceeds to higher masses, and there are a number of reactions requiring more precise study [20]. After hydrogen burning, helium and then carbon burning ensues. The relevant reactions of helium burning are still not understood on a sufficient level of precision [21]. This applies, e.g., to the ${ }^{12} \mathrm{C}(\alpha, \gamma){ }^{16} \mathrm{O}$, ${ }^{16} \mathrm{O}(\alpha, \gamma){ }^{20} \mathrm{Ne}$, and ${ }^{18} \mathrm{O}(\alpha, \gamma){ }^{22} \mathrm{Ne}$ reactions. Similar considerations are valid for the reactions of carbon burning $[22]$, e.g. ${ }^{12} \mathrm{C}\left({ }^{12} \mathrm{C}, \mathrm{p}\right){ }^{23} \mathrm{Na}$, and for the neutron source reactions powering the astrophysical s-process.

All these cases involve light, stable nuclei as beams and targets, and the in-beam detection of $\gamma$-rays or neutrons. Therefore, they can be studied at ion accelerator facilities with a few MV accelerating potential and sufficient beam intensity, provided the laboratory background is low enough. Indeed, there is a call for new underground accelerators $[23,24]$. Relevant projects have been proposed in deep-underground settings, below a rock overburden of $1000 \mathrm{~m}$ or more $[11,24,25]$.

It is shown here that already shallow-underground sites present satisfactory background conditions for a number of nuclear astrophysics experiments, if a suitable active shield, surrounding either just the detector or the whole laboratory, suppresses remaining muons. As a demonstration, a study of background in $\gamma$-ray detectors in the Felsenkeller shallow-underground laboratory (47 $\mathrm{m}$ rock overburden) in Dresden (Germany) has been carried out.

For underground accelerator-based experiments, the $Q$-value of a typical radiative capture reaction is usually much larger than the beam energy $E$ in the centerof-mass system. Therefore, the $\gamma$-ray energy for capture to the ground state $E_{\gamma}=Q+E$ (neglecting the small Doppler and recoil corrections) is usually dominated by the $Q$-value, and in many cases $E_{\gamma}>2.615 \mathrm{MeV}$. The background and the feasibility of radiative capture experiments strongly depend on the $\gamma$-ray energy.

In sect. 2 , the background for $E_{\gamma} \leq 2.615 \mathrm{MeV}$ is reviewed at different sites, based on literature data. The main characteristics of the Felsenkeller shallowunderground facility included in this background comparison are described in sect. 3. In sect. 4 and 5 , a background comparison between the Earth's surface, shallow-, and deep-underground sites is performed by subsequently transporting one and the same detector to each of the three sites. The implications for the feasibility of experiments using simple single-detector setups underground are discussed in sect. 6 , and a summary is given in sect. 7 .

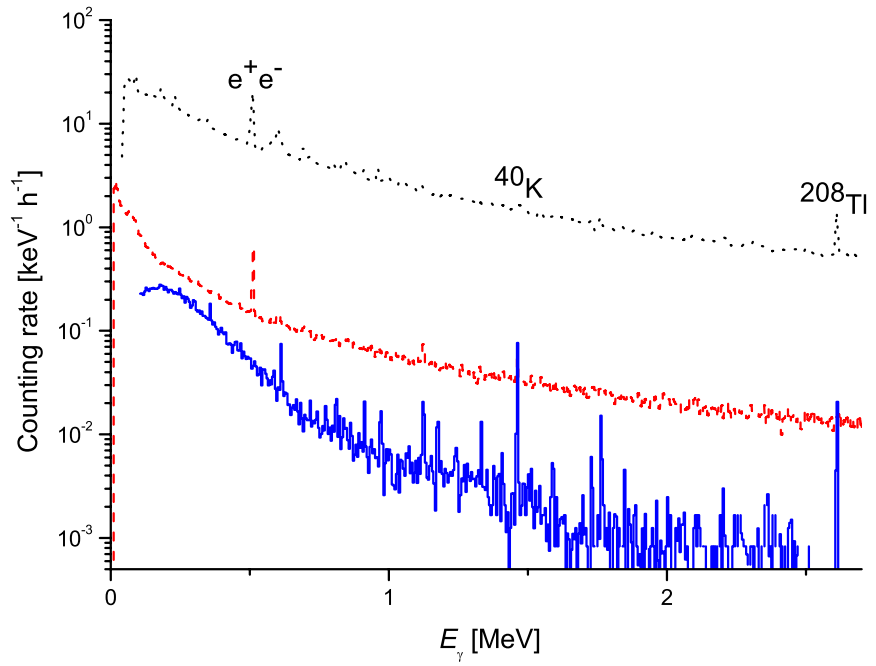

Fig. 1. (Color online) Review of previous background data using HPGe detectors without active shielding, but with full lead and copper shield. The data are rescaled to match $122 \%$ relative efficiency. Earth's surface (black dotted line [26]), shallow underground (red dashed line [27]), deep underground (blue full line [9]).

\section{Review of previous data on the background in passively shielded germanium detectors at several depths}

Before turning to the new experimental data obtained here, in the present section the background situation in detectors without active shielding underground is briefly reviewed based on the literature. The discussion concentrates on $\gamma$-energies below the $2.615 \mathrm{MeV}$ line of ${ }^{208} \mathrm{Tl}$, where abundant data are available [4]. Data from nuclear astrophysics related setups are selected for this comparison, where possible.

At these $\gamma$-energies, a thick lead shield is essential to suppress the background from radionuclides. However, an additional rock overburden also helps reduce the continuum component of the background [4]. Published data from three well-shielded p-type HPGe detector setups, each of them with the optimal shielding configuration for its depth [4], are shown to illustrate this point:

1) The first HPGe detector is hosted in a $15 \mathrm{~cm}$ thick lead shield at the Earth's surface [26]. For surface-based experiments, $15 \mathrm{~cm}$ is the optimum shielding thickness, as the background does not decrease significantly when using even thicker shields [4].

2) The second HPGe detector is contained in a shield of $15 \mathrm{~cm}$ lead and $5 \mathrm{~cm}$ copper shallow underground at Felsenkeller [27] (see also sect. 3 of the present work).

3) The third one is hosted in a shield of $25 \mathrm{~cm}$ lead and $4 \mathrm{~cm}$ copper deep underground at Gran Sasso, in a setup dedicated to ultra-low background in-beam $\gamma$ spectrometry [9].

The relative efficiencies of the three detectors are of the same order of magnitude (100\% [26], 90\% [27], 137\% [9]). 


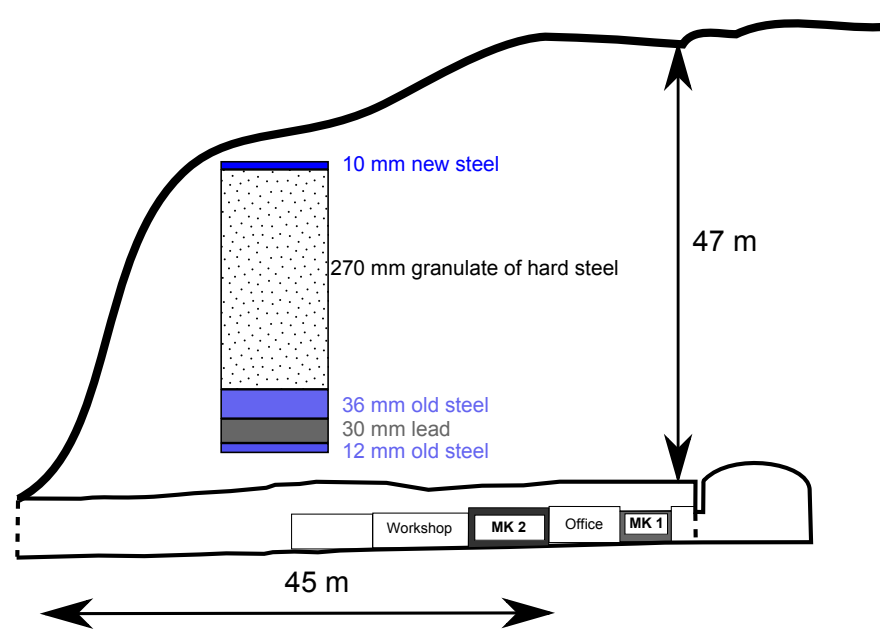

Fig. 2. Cross-section of tunnel IV of the Felsenkeller underground facility. The current underground counting facility with its two measuring chambers MK1 [28] and MK2 [29] is also shown. The inset shows the makeup of the shielding of measuring chamber MK2, from the outside (top) to the inside (bottom).

In order to facilitate a quantitative comparison between their background levels, the counting rates from the three detectors are rescaled to match $122 \%$ relative efficiency, the efficiency of the HPGe detector used in sect. 5 .

From this limited review of published data, concentrating on nuclear astrophysics related setups, it can be seen that for $0.100 \mathrm{MeV} \leq E_{\gamma} \leq 2.615 \mathrm{MeV}$, the background shallow underground is a factor 40 lower than at the Earth's surface, and again a factor 3-20 lower deep underground (fig. 1). As a consequence, passively shielded shallow-underground facilities may provide some kind of intermediate solution between surface-based and deep-underground experiments, when it comes to in-beam $\gamma$-spectrometric experiments for nuclear astrophysics with detected $\gamma$-rays of $E_{\gamma}<2.615 \mathrm{MeV}$.

\section{The Felsenkeller site and laboratory}

The Felsenkeller area is located in an ancient quarry in the Weißeritz valley, $5 \mathrm{~km}$ from Dresden city center. The underground facility consists of nine tunnels, called tunnel I-IX, that have been dug in the 1850's to host the ice cellar of the nearby Felsenkeller brewery.

A low-background radioactivity counting facility was installed in 1982 in tunnel IV [28], with a measuring chamber called MK1 that was shielded by a low-radioactivity serpentinite rock. In 1995, a new measuring chamber called MK2 has been added to the facility [29]. The walls of MK2 are $36 \mathrm{~cm}$ thick, in part consisting of old, ${ }^{60} \mathrm{Co}$-free steel from an epoch predating the nuclear age (fig. 2). The average rock overburden above the VKTA facility is $47 \mathrm{~m}$, equivalent to $110 \mathrm{~m}$ water (m.w.e.), leading to a reduction of the muon flux by a factor of 30-50 depending on the angle being studied.
The analytics facility of tunnel IV, operated by VKTA Dresden, is a founding member of the European CELLAR Collaboration of underground low-background laboratories. It hosts several HPGe $\gamma$-detectors and a tritium counting facility. Recently a new ultra-low background germanium detector made of specially selected components went into operation [27]. In recent years, also nuclear astrophysics [30] and rare nuclear decay [31] studies have been performed at Felsenkeller.

Tunnels I-III and V-IX are used as storage sites by local businesses. They have a typical diameter of $6 \mathrm{~m}$ and the same rock overburden as tunnel IV.

\section{New data using a "traveling" $\mathrm{LaBr}_{3}$ detector}

In order to extend the background comparison performed in sect. 2 to higher $\gamma$-energies, $2.615 \mathrm{MeV}<E_{\gamma}$, two new experiments have been performed ${ }^{1}$. In both cases, one and the same detector has been used subsequently at the three sites Earth's surface, shallow underground, and deep underground. By using exactly the same device, any observed background differences can be attributed to the characteristics of the laboratory, not the ones of the specific detector.

The shallow-underground data for the comparison have been recorded in the MK2 chamber of Felsenkeller (sect. 3). Deep-underground data have been taken in the Gran Sasso laboratory in Italy (1400 m rock overburden). The comparison data at the Earth's surface have been taken in the basement of a three-story building on the HZDR Rossendorf campus. In the present section, the measurements with a lanthanum bromide $\left(\mathrm{LaBr}_{3}\right)$ detector are presented. Data with a high-purity germanium (HPGe) detector are shown in the subsequent sect. 5 .

The $\mathrm{LaBr}_{3}$ detector had a cylindrical shape with $6.5 \mathrm{~cm}$ length, $8.3 \mathrm{~cm}$ diameter, and $350 \mathrm{~cm}^{3}$ volume. For the measurements at the Earth's surface and at Felsenkeller, it was inserted in the center of a large BGO anticompton shield of $23 \mathrm{~cm}$ length that had been designed for the use with a EUROBALL Cluster detector.

Up to the $2.615 \mathrm{MeV}$ line of ${ }^{208} \mathrm{Tl}$, the $\mathrm{LaBr}_{3}$ spectrum (fig. 3) is dominated by the intrinsic ${ }^{138}$ La activity of $\mathrm{LaBr}_{3}$ and by quenched $\alpha$-particles from the decay of its intrinsic ${ }^{227}$ Ac contamination [32]. Consequently, at these $\gamma$-energies the observed counting rate does not depend on the rock overburden at the laboratory. In the range from $2.615 \mathrm{MeV}$ up to $5 \mathrm{MeV}$, the effect of pileup of $\alpha$-emitters in the crystal is visible, which again leads to a sizable depth-independent contribution to the counting rate. Because of these effects, only the part of the $\mathrm{LaBr}_{3}$ spectra above $5 \mathrm{MeV}$ is used for the comparison, where ${ }^{138} \mathrm{La}$, natural radionuclides, and $\alpha$-emitters in the crystal do not play a role.

\footnotetext{
${ }^{1}$ In this work, the symbol $E_{\gamma}$ is used throughout to denote the $\gamma$-ray equivalent energy at which the background is registered in the detector under study.
} 


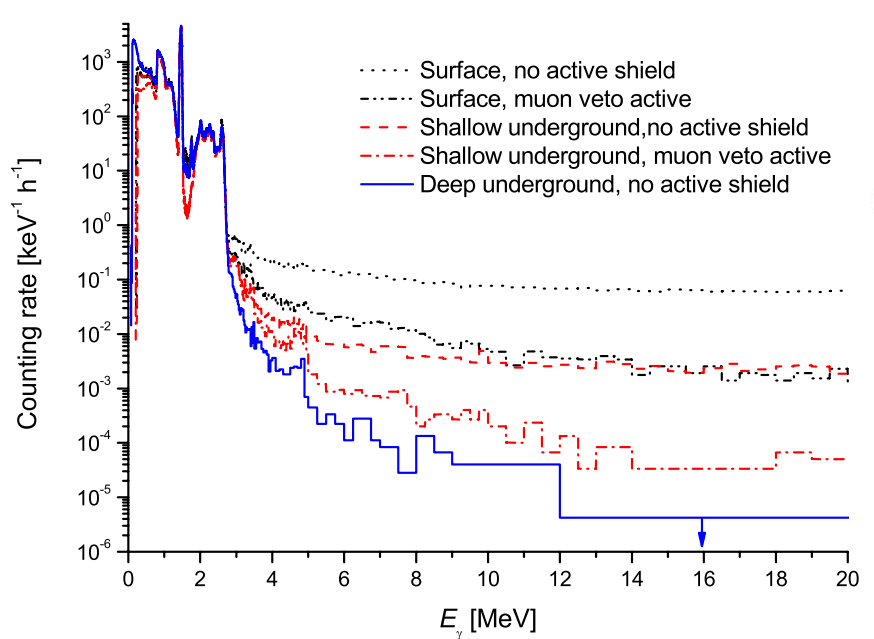

Fig. 3. (Color online) Background in one and the same actively shielded $\mathrm{LaBr}_{3}$ detector: Earth's surface, without (black dotted line) and with (black dot-dot-dashed line) active muon veto. Shallow underground, without (red dashed line) and with (red dot-dashed line) active muon veto. Deep underground without active muon veto (blue solid line).

For $5 \mathrm{MeV}<E_{\gamma}<12 \mathrm{MeV}$, the counting rate at the Earth's surface is dominated by the energy loss of passing or stopping muons, and by the capture of energetic neutrons created by muons in material surrounding the detector. Both these effects are attenuated in the shallowunderground laboratory Felsenkeller (red dashed line in fig. 3), where the muon flux is reduced by a factor of 30 . The remaining muons, but not the neutrons produced by them, are suppressed further by applying the active shield (red dot-dashed line in fig. 3).

Deep underground, the muon flux is a factor $10^{6}$ lower than at the surface, but the counting rate in the $5 \mathrm{MeV}<$ $E_{\gamma}<12 \mathrm{MeV}$ region is only a factor $10^{3}$ lower. This suppression factor of $10^{3}$ is consistent with previous work [8, 10]. It is due to the fact that a depth-independent flux of high-energy neutrons created by $(\alpha, \mathrm{n})$ reactions and spontaneous fission of ${ }^{238} \mathrm{U}$ in the rock [33] dominates the deepunderground background. A neutron shield would help here, but the counting rates deep underground are already sufficiently low without it [8]. For the deep-underground run with this detector, no active shield was used.

For $12 \mathrm{MeV}<E_{\gamma}$, neutrons play less of a role, and the effect of the muon flux reduction with depth and/or active shield is well visible. No counts were observed in $30 \mathrm{~h}$ counting time in the deep-underground spectrum in this energy range, so the given value for $12 \mathrm{MeV}<E_{\gamma}$ is a $1 \sigma$ upper limit.

\section{New data using a "traveling" HPGe detector}

The second "traveling" detector was a Clover-type HPGe detector of $7.1 \mathrm{~cm}$ length, with a tapered front face of $8.2-9.1 \mathrm{~cm}$ sidelength and $470 \mathrm{~cm}^{3}$ volume. It is configured in addback mode [34] (122\% relative efficiency) and

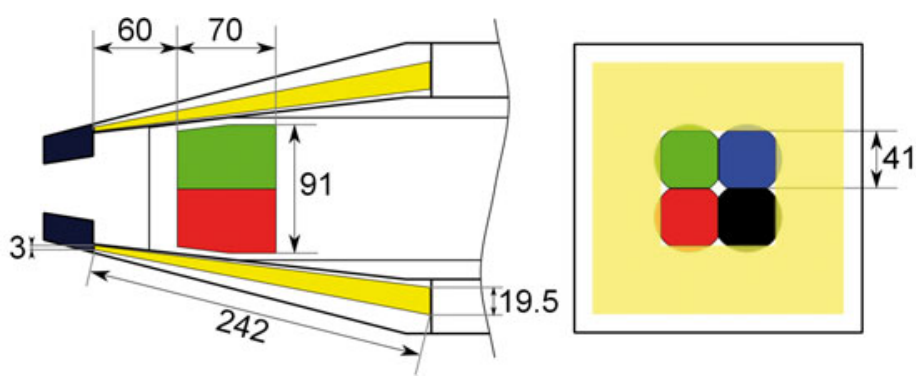

Fig. 4. (Color online) Schematic view of the HPGe detector (four sections: green, red, black, and blue) and its BGO escapesuppression shield (yellow) that were used for the present study. Dimensions are given in $\mathrm{mm}$. The four individual crystals have a tapered cylindrical shape. Left panel: View from the side. Right panel: Front view.

used in anticoincidence with a surrounding BGO scintillator of $24 \mathrm{~cm}$ length (fig. 4). The BGO escape suppression thus forms also an efficient muon veto. Further details on this particular detector are included in a previous publication [10]. In that paper, the deep-underground spectra of this device have already been published [10].

For the purposes of the present work, additional runs were maken with the same detector in the Felsenkeller shallow-underground laboratory, and at the Earth's surface in the basement of a three-story building on the HZDR Rossendorf campus. The surface-based data showed the so-called muon peak [35] at $50-60 \mathrm{MeV}$ in this detector's spectrum, roughly the energy expected for its size. This peak is suppressed by a factor of 160 by the BGO anticoincidence, showing how efficiently this suppression works.

This detector was then transported to Felsenkeller (spectrum in fig. 5). Naturally occurring radionuclides dominate the background up to the $2.615 \mathrm{MeV}$ line of ${ }^{208} \mathrm{Tl}$. Note that in this energy region and in this detector, the shallow-underground background is even lower than the deep-underground one. This surprising fact is due to the thick iron and lead walls of the MK2 measuring chamber in Felsenkeller, whereas no similar shielded chamber was used at Gran Sasso. For the $\mathrm{LaBr}_{3}$ detector discussed in sect. 4, the effect of the MK2 walls was less striking due to its higher intrinsic background. Still, for an inter-laboratory comparison the data with the traveling HPGe and $\mathrm{LaBr}_{3}$ detectors should only be used above the $2.615 \mathrm{MeV}$ line of ${ }^{208} \mathrm{Tl}$.

As in the case of the $\mathrm{LaBr}_{3}$ detector, in order to completely exclude depth-independent effects such as the MK2 walls and intrinsic $\alpha$-emitters from a ${ }^{210} \mathrm{Po}$ contamination [36], the comparison should be limited to the highenergy region, $5.3 \mathrm{MeV} \leq E_{\gamma}$. Indeed, the effect of the $\mathrm{BGO}$ active shield is best seen in this region, where muoninduced effects dominate.

For $5.3 \mathrm{MeV} \leq E_{\gamma} \leq 8.0 \mathrm{MeV}$, the counting rate without active shield is dominated by the energy loss of passing muons, both in the surface-based and in the shallowunderground runs. The ratio of the surface to the shallowunderground data is a factor of 40 , the same as the sup- 


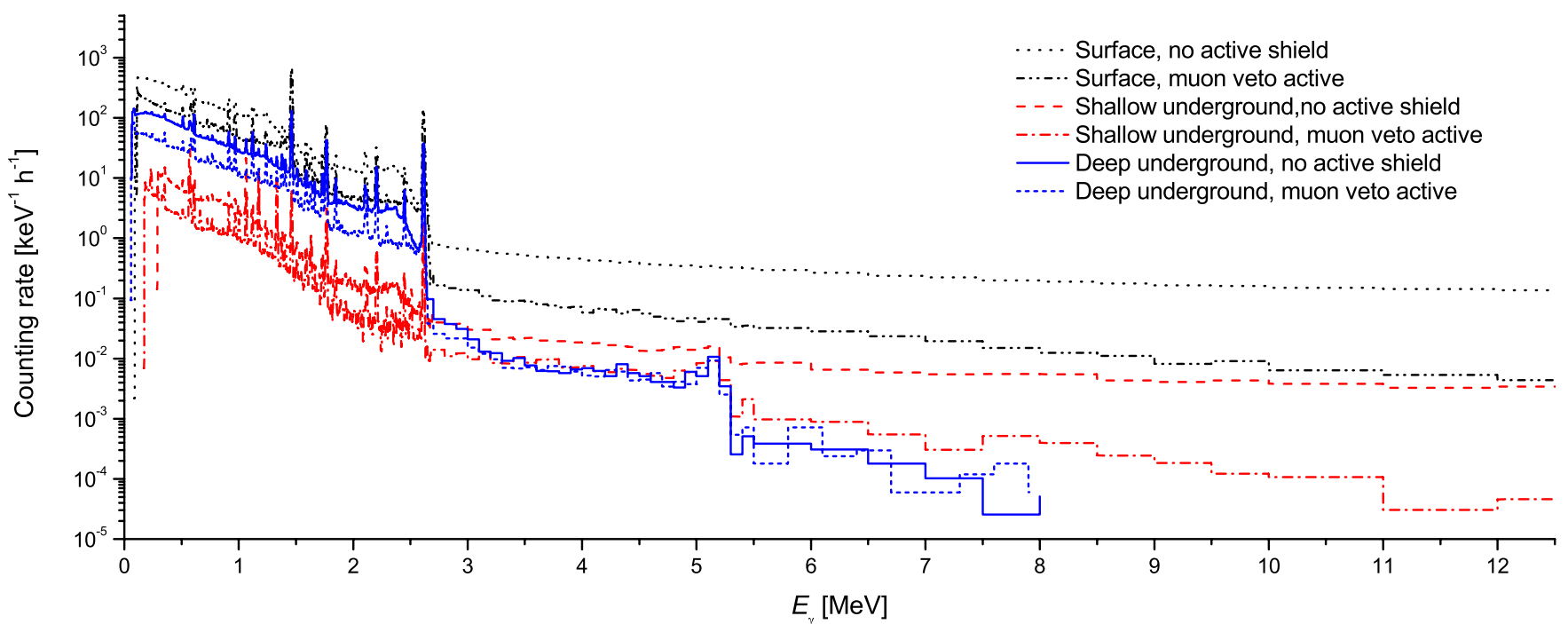

Fig. 5. (Color online) Background in one and the same Clover HPGe detector: Earth's surface, without (black dotted line) and with active muon veto (black dot-dot-dashed line). Shallow underground, without (red dashed line) and with active muon veto (red dot-dashed line). Deep underground [10] (limited to $0-8 \mathrm{MeV}$ ), without (blue solid line) and with active muon veto (blue short dashed line).

pression of the muon flux. Deep underground, an additional factor of $3 \times 10^{4}$ suppression is expected for the direct effects of muons, bringing this source of background to a negligible level. Instead, as discussed above, again depthindependent neutrons emitted from the walls of the laboratory due to $(\alpha, \mathrm{n})$ reactions dominate the counting rate. These neutrons are not affected by the escape-suppression shield, so the deep-underground counting rate with and without muon veto is the same in this energy region [10].

As a result, for $5.3 \mathrm{MeV} \leq E_{\gamma} \leq 8.0 \mathrm{MeV}$ the counting rate in one and the same detector deep underground is only a factor $2.4 \pm 0.3$ lower than shallow underground, if an active muon veto is used. Due to the dynamic range of the deep-underground data for this particular detector, no direct comparison is possible for $8.0 \mathrm{MeV}<E_{\gamma}$, but the trend can be estimated from the $\mathrm{LaBr}_{3}$ data, which extend to higher energies.

\section{Implications for the feasibility of accelerator-based experiments}

In order to gauge the feasibility of accelerator-based experiments on radiative capture reactions, some real or hypothetical setup has to be assumed. Depending on whether the $\gamma$-rays to be detected lie outside the range of natural radionuclides $\left(E_{\gamma}>2.615 \mathrm{MeV}\right)$ or inside it $\left(E_{\gamma}\right.$ $\leq 2.615 \mathrm{MeV})$, different shielding scenarios are optimal, therefore for those two $\gamma$-energy ranges two different setups called setup A and B, respectively, are assumed.

Setup A: For reactions with emitted $\gamma$-rays of $E_{\gamma}>$ $2.615 \mathrm{MeV}$, as discussed above $\gamma$-lines from environmental radionuclides do not play a role, and a lead shield around the detector or around the whole counting room as in the MK2 case cannot be expected to further improve the background. Therefore, for these reactions a setup used previously for in-beam $\gamma$-spectroscopy experiments at LUNA $[16,10,17]$ is selected for the feasibility discussion. Setup A consists of the present Clover HPGe detector (sect. 5), with its endcap at $9.5 \mathrm{~cm}$ distance from the target and the present active BGO veto. For this setup, a typical random veto rate of $1 \%$ due to background hitting the BGO has been found [10]. For $\gamma$-rays emitted in cascade, a typical peak suppression of $5 \%$ has been observed due to the second $\gamma$-ray of a cascade hitting the BGO shield $[16,17]$. The latter effect vanishes for a single emitted $\gamma$-ray, as in capture to the ground state of the compound nucleus.

Setup B: For reactions with emitted $\gamma$-rays of $E_{\gamma}<$ $2.615 \mathrm{MeV}$, a lead shield is necessary to suppress environmental $\gamma$-lines. For studying these cases, the present $\mathrm{LaBr}_{3}$ and HPGe data (sect. 4 and 5) cannot be used due to the lack of proper lead shielding. It would have been prohibitively difficult to transport not only the detectors, but also one and the same full lead shield to the above discussed three sites. For the cases with $E_{\gamma}<2.615 \mathrm{MeV}$, instead the previous background data from well-shielded setups (sect. 2) are used, rescaled for their relative efficiencies as stated above (fig. 1). The same detector-target distance as in setup A is assumed. This hypothetical setup is called setup B.

A typical target of $6 \cdot 10^{17}$ active target atoms $/ \mathrm{cm}^{2}$ is assumed, with the composition given in table 1. The $S$-factor or resonance strength and branching ratio are adopted from the given reference (table 1 ). A beam intensity of 250 particle- $\mu \mathrm{A}$ is assumed.

For the cases considered here, one or more $\gamma$-rays are emitted per reaction. However, in many cases including most of the examples given here, capture to the ground state dominates at the energies under study, or is even 
Table 1. Signal counting rate in a single-detector setup for capture to the ground state of the final nucleus, at the center-of-mass energy $E$. For ${ }^{12} \mathrm{C}$ in $\mathrm{Au}$, a ratio of $5: 1$ [37] is assumed. See the text for further details. The background counting rate at different depths is also given.

\begin{tabular}{|c|c|c|c|c|c|c|c|c|c|c|}
\hline Reaction & $E$ & Ref. & $\gamma$-ray ROI & Target & Setup & Full energy peak & Signal & \multicolumn{3}{|c|}{ Laboratory background counting rate $\left[\mathrm{h}^{-1}\right]$} \\
\hline & {$[\mathrm{keV}]$} & & {$[\mathrm{keV}]$} & & & $\gamma$-efficiency & {$\left[\mathrm{h}^{-1}\right]$} & Earth's surface & Shallow undergr. & Deep undergr. \\
\hline${ }^{12} \mathrm{C}\left({ }^{12} \mathrm{C}, \mathrm{p}\right){ }^{23} \mathrm{Na}\left({ }^{\mathrm{a}}\right)$ & 2200 & {$[22]$} & $438-441$ & ${ }^{12} \mathrm{C}$ in $\mathrm{Au}$ & B & $9.0 \times 10^{-3}$ & 0.80 & $20.3 \pm \quad 0.5$ & $0.53 \pm 0.02$ & $0.179 \pm 0.018$ \\
\hline${ }^{3} \mathrm{He}(\alpha, \gamma){ }^{7} \mathrm{Be}$ & 200 & {$[38]$} & 1779-1789 & ${ }^{3} \mathrm{He}$ gas & B & $3.9 \times 10^{-3}$ & 190 & $10.5 \pm \quad 0.4$ & $0.250 \pm 0.016$ & $0.010 \pm 0.004$ \\
\hline${ }^{12} \mathrm{C}(\mathrm{p}, \gamma){ }^{13} \mathrm{~N}$ & 80 & {$[39]$} & $2009-2027$ & ${ }^{12} \mathrm{C}$ in $\mathrm{Au}$ & B & $3.5 \times 10^{-3}$ & 0.52 & $14.6 \pm \quad 0.4$ & $0.39 \pm 0.02$ & $0.017 \pm 0.005$ \\
\hline${ }^{16} \mathrm{O}(\alpha, \gamma){ }^{20} \mathrm{Ne}$ & 1300 & {$[40]$} & 5991-6035 & $\mathrm{Al}_{2} \mathrm{O}_{3}$ & A & $1.2 \times 10^{-3}$ & 0.025 & $1.39 \pm 0.05$ & $0.039 \pm 0.007\left(^{\mathrm{b}}\right)$ & $0.021 \pm 0.006$ \\
\hline${ }^{12} \mathrm{C}(\alpha, \gamma){ }^{16} \mathrm{O}$ & 800 & {$[21]$} & 7929-7968 & ${ }^{12} \mathrm{C}$ in $\mathrm{Au}$ & A & $8.9 \times 10^{-4}$ & 0.018 & $0.59 \pm 0.05$ & $0.024 \pm 0.008\left(^{\mathrm{b}}\right)$ & $0.006 \pm 0.004$ \\
\hline$\left.{ }^{18} \mathrm{O}(\alpha, \gamma)\right)^{22} \mathrm{Ne}$ & 385 & {$[41]$} & $10045-10058$ & $\mathrm{Al}_{2}{ }^{18} \mathrm{O}_{3}$ & A & $6.7 \times 10^{-4}$ & 0.045 & $0.082 \pm 0.005$ & $0.0015 \pm 0.0006\left(^{\mathrm{b}}\right)$ & $0.0006 \pm 0.0004\left({ }^{\mathrm{c}}\right)$ \\
\hline${ }^{15} \mathrm{~N}(\mathrm{p}, \gamma){ }^{16} \mathrm{O}$ & 70 & {$[42]$} & $12163-12205$ & $\mathrm{Ti}^{15} \mathrm{~N}$ & A & $5.3 \times 10^{-4}$ & 0.012 & $0.188 \pm 0.013$ & $0.0019 \pm 0.0011\left(^{\mathrm{b}}\right)$ & $<0.0002\left({ }^{\mathrm{c}}\right)$ \\
\hline
\end{tabular}

$\left({ }^{\mathrm{a}}\right)$ Decay of the first excited state of ${ }^{23} \mathrm{Na}$.

( $\left.{ }^{b}\right)$ Consistent background is found with the present Clover HPGe detector in an unshielded part of the Felsenkeller facility outside MK2.

( ${ }^{\mathrm{C}}$ ) Rescaled from the $\mathrm{LaBr}_{3}$ background.

the only significant reaction mechanism. For these cases, the region of interest (ROI) width has been determined from the energetic target thickness, folded with the resolution. For the fixed-energy $\gamma$-rays from the ${ }^{12} \mathrm{C}\left({ }^{12} \mathrm{C}, \mathrm{p}\right){ }^{23} \mathrm{Na}$ and ${ }^{18} \mathrm{O}(\alpha, \gamma)^{22} \mathrm{Ne}$ reactions, the width of the $\gamma$-ray ROI is taken as two times the full width at half maximum (FWHM) $\gamma$-energy resolution.

It should be noted that the given background levels are a lower limit, neglecting ion beam induced background. However, this latter problem usually did not limit experiments at LUNA [11].

In principle, an experiment is also possible when the signal is much lower than the background. However, the time required to reach a given statistics scales with the ratio of background to signal. The running times for the type of experiment discussed here are of the order of weeks or even months per data point, so that an experiment cannot be concluded in a realistic time when the background is much higher than the signal. Therefore, the signal counting rate should be higher than or at least comparable to the background in order for experiments to be feasible.

When adopting this criterion, it is apparent that for five of the reactions listed, an experiment at the surface of the Earth is impossible. For the remaining two, namely ${ }^{3} \mathrm{He}(\alpha, \gamma){ }^{7} \mathrm{Be}$ and ${ }^{18} \mathrm{O}(\alpha, \gamma){ }^{22} \mathrm{Ne}$, recent experiments at the Earth's surface either did not reach low energies [43] or were still limited by background [41].

When comparing the background values at the three sites listed in the right three colums of table 1 , two observations can be made. First, for all the cases studied here, the shallow-underground scenario offers already a background that is a factor of 25-100 lower than at the Earth's surface, sufficiently low for the experiments to be feasible. Second, the background in deep underground is even lower than that in shallow underground: In the vetoed setup A for $5.3 \mathrm{MeV}<E_{\gamma}<8.0 \mathrm{MeV}$, by a factor of $2.4 \pm 0.3$. In the non-vetoed setup B for $1.5 \mathrm{MeV}<E_{\gamma}<2.7 \mathrm{MeV}$, by a factor of $19 \pm 1$. A smaller difference is observed at lower energy, $E_{\gamma} \approx 0.4 \mathrm{MeV}$, where bremsstrahlung from ${ }^{210} \mathrm{Bi}$ dominates the particular deep-underground spectrum [9] used for the comparison.

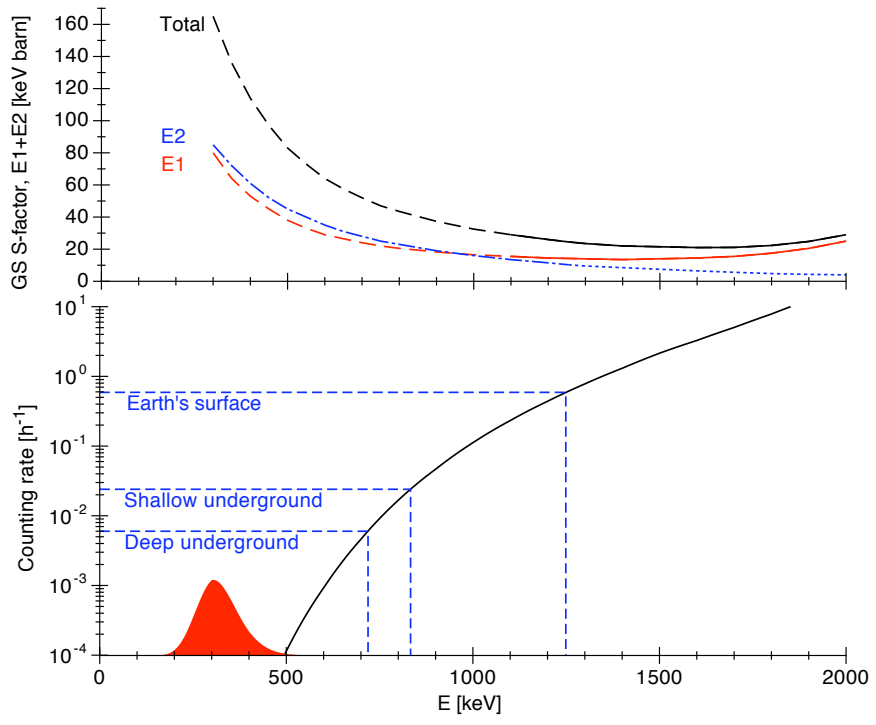

Fig. 6. (Color online) Top panel: Astrophysical $S$-factor for ground-state capture in ${ }^{12} \mathrm{C}(\alpha, \gamma){ }^{16} \mathrm{O}$, from $R$-matrix fits for its $E 1$ [21] and $E 2$ [44] contributions. The energy range with (without) experimental cross-section data is indicated by a full (dashed) line. Bottom panel: Predicted ${ }^{12} \mathrm{C}(\alpha, \gamma){ }^{16} \mathrm{O}$ counting rate in the present single-detector setup $\mathrm{A}$, compared to the background in the various sites. The Gamow peak for $T_{9}=0.2$ is also given, in linear scale.

In order to illustrate the gain in sensitivity when moving from the Earth's surface to the different underground scenarios, the ${ }^{12} \mathrm{C}(\alpha, \gamma){ }^{16} \mathrm{O}$ reaction is selected. At astrophysical energies, this reaction is believed to be dominated by capture to the ground state in ${ }^{16} \mathrm{O}[21]$, and for this transition both high-energy experimental data and $R$ matrix fits $[44,21]$ exist. There is about $30 \%$ uncertainty in the extrapolated $S$-factor [21], whereas stellar modelers call for $10 \%$ precision [45]. Taking advantage of the lower background, it is possible to approach the Gamow peak significantly in a shallow underground setting (fig. 6), halving the distance between the energies where data exist and the astrophysical energy. 
The data also show that deep underground, it is possible to go even further down in energy, closer to the Gamow peak. For the single-detector scenario considered here, the counting rates would then become very low, less than one count per week. As a consequence, probably much larger detector arrays should be considered to fully exploit the advantages of working deep underground.

For a large $\gamma$-detector array, the present escape-suppression scheme of a BGO veto detector immediately surrounding each germanium crystal is not practical any more. Instead, a muon veto can in principle be achieved in two different ways: By covering the outside of the large detector array with a veto detector that is many square meters large [46], or by using many closely packed germanium detectors that function as a veto detector for each other, using $\gamma$-ray tracking and pulse shape discrimination techniques [47].

A detailed study of how a large detector array with an appropriate muon veto, which is complicated and costly to build, would function in a deep- or shallow-underground setting is beyond the scope of the present work. Instead, here the feasibility discussion is limited to setups of much lower complexity and cost, including just one detector, and comparing one and the same detector in different sites.

\section{Summary}

A dedicated study of the background in a standard inbeam $\gamma$-spectroscopy setup has been performed at the Earth's surface and in shallow- and deep-underground facilities. Active shielding has been used to suppress the direct effects of cosmic-ray muons. Based on the data, the feasibility of accelerator-based nuclear astrophysics experiments has been evaluated for several key cases, in a singledetector setup.

It has been shown that experiments at the surface of the Earth are not a realistic option for any of the reactions considered here. Instead, an underground setting is needed. Owing to the fact that nuclear reaction experiments require low but not ultra-low background (e.g., [48]), the present data show that already shallowunderground sites offer satisfactory background conditions for a number of in-beam $\gamma$-spectrometry experiments in a single-detector setup. It was also shown that even better background conditions are reached deep underground.

This finding opens the door for a complementary approach to place our understanding of stellar nucleosynthesis on a firm experimental foundation, much the same way as has been achieved with fusion in the Sun: The ease of access to shallow-underground facilities and standard detectors should be exploited to quickly gain low-energy data near the Gamow peak, while large detector arrays in deep-underground sites push the data limit even lower, in some cases to astrophysical energies.

This work was supported in part by the Herbert Quandt Foundation, DFG (BE 4100/2-1), OTKA (K101328, NN83261), TÁMOP-4.2.2/B-10/1-2010-0024, EuroGENESIS, and the EU (European Social Fund).
Open Access This is an open access article distributed under the terms of the Creative Commons Attribution License (http://creativecommons.org/licenses/by/2.0), which permits unrestricted use, distribution, and reproduction in any medium, provided the original work is properly cited.

\section{References}

1. C. Rolfs, W. Rodney, Cauldrons in the Cosmos (University of Chicago Press, Chicago, 1988).

2. C. Iliadis, Nuclear Physics of Stars (Wiley-VCH, Weinheim, 2007).

3. R.N. Boyd, An introduction to Nuclear Astrophysics (The University of Chicago Press, Chicago, 2008).

4. G. Heusser, Annu. Rev. Nucl. Part. Sci. 45, 543 (1995).

5. J.A. Formaggio, C. Martoff, Annu. Rev. Nucl. Part. Sci. 54, 361 (2004).

6. A. da Silva et al., Nucl. Instrum. Methods A 354, 553 (1995).

7. D. Mei, A. Hime, Phys. Rev. D 73, 053004 (2006).

8. D. Bemmerer et al., Eur. Phys. J. A 24, 313 (2005).

9. A. Caciolli et al., Eur. Phys. J. A 39, 179 (2009).

10. T. Szücs et al., Eur. Phys. J. A 44, 513 (2010).

11. C. Broggini, D. Bemmerer, A. Guglielmetti, R. Menegazzo, Annu. Rev. Nucl. Part. Sci. 60, 53 (2010).

12. R. Bonetti et al., Phys. Rev. Lett. 82, 5205 (1999) arXiv:nucl-ex/9902004.

13. A. Formicola et al., Phys. Lett. B 591, 61 (2004).

14. A. Lemut et al., Phys. Lett. B 634, 483 (2006).

15. D. Bemmerer et al., Phys. Rev. Lett. 97, 122502 (2006).

16. M. Marta et al., Phys. Rev. C 78, 022802(R) (2008).

17. M. Marta et al., Phys. Rev. C 83, 045804 (2011) arxiv:1103.5393.

18. E. Adelberger et al., Rev. Mod. Phys. 83, 195 (2011) arXiv:1004.2318.

19. F. Iocco, G. Mangano, G. Miele, O. Pisanti, P.D. Serpico, Phys. Rep. 472, 1 (2009) arxiv:0809.0631.

20. M. Wiescher, J. Görres, E. Uberseder, G. Imbriani, M. Pignatari, Annu. Rev. Nucl. Part. Sci. 60, 381 (2010).

21. L. Buchmann, C. Barnes, Nucl. Phys. A 777, 254 (2006).

22. T. Spillane et al., Phys. Rev. Lett. 98, 122501 (2007).

23. The DOE/NSF Nuclear Science Advisory Committee, The Frontiers of Nuclear Science, A Long Range Plan (DOE/NSF Nuclear Science Advisory Committee, 2008), arXiv:0809.3137.

24. Nuclear Physics European Collaboration Committee, Long Range Plan: Perspectives of Nuclear Physics in Europe (NuPECC, 2010).

25. A. Lemut et al., Phys. Rev. ST Accel. Beams 14, 100101 (2011).

26. M. Erhard, Photoaktivierung des p-Kerns ${ }^{92}$ Mo am Bremsstrahlungsmessplatz von ELBE, PhD thesis, TU Dresden (2009).

27. M. Köhler et al., Appl. Radiat. Isot. 67, 736 (2009).

28. W. Helbig, S. Niese, D. Birnstein, Isotopenpraxis 20, 60 (1984).

29. S. Niese, M. Köhler, B. Gleisberg, J. Radioanal. Nucl. Chem. 233, 167 (1998).

30. C. Nair et al., Phys. Rev. C 81, 055806 (2010) arxiv:1005.2158.

31. J. Dawson et al., Phys. Rev. C 78, 035503 (2008) arxiv:0804.1198. 
32. F. Crespi et al., Nucl. Instrum. Methods A 602, 520 (2009)

33. H. Wulandari, J. Jochum, W. Rau, F. von Feilitzsch, Astropart. Phys. 22, 313 (2004).

34. G. Duchêne et al., Nucl. Instrum. Methods A 432, 90 (1999).

35. R. Wordel et al., Nucl. Instrum. Methods A 369, 557 (1996).

36. R.L. Brodzinski, J.H. Reeves, F.T. Avignone, H.S. Miley, Nucl. Instrum. Methods A 254, 472 (1987).

37. S. Seuthe et al., Nucl. Instrum. Methods A 260, 33 (1987).

38. F. Confortola et al., Phys. Rev. C 75, 065803 (2007).

39. C. Angulo et al., Nucl. Phys. A 656, 3 (1999).

40. H. Costantini et al., Phys. Rev. C 82, 035802 (2010).
41. S. Dababneh et al., Phys. Rev. C 68, 025801 (2003).

42. D. Bemmerer et al., J. Phys. G 36, 045202 (2009).

43. T.A.D. Brown et al., Phys. Rev. C 76, 055801 (2007) arxiv:0710.1279.

44. R. Kunz et al., Phys. Rev. Lett. 86, 3244 (2001).

45. S.E. Woosley, A. Heger, Phys. Rep. 442, 269 (2007) arXiv:astro-ph/0702176.

46. B. Bodmann et al., Nucl. Instrum. Methods A 286, 214 (1990).

47. E. Farnea et al., Nucl. Instrum. Methods A 621, 331 (2010).

48. B. Aharmim et al., Phys. Rev. Lett. 101, 111301 (2008) arxiv:0806.0989. 